\title{
About the influence of temperature and humidity level on pulse shape parameters of positive DC corona discharges in air
}

\author{
Ulrich Lühring, Daniel Wienold and Frank Jenau
}

\begin{abstract}
Partial discharges reduce the residual lifespan of operating equipment and can indicate serious deficiencies of electrical insulations at an early stage. Regarding AC voltage stress, the phase resolved pattern is an established diagnostic tool, which allows conclusions about the location and the type of fault. Despite the increasing importance of DC transmission systems, a comparable proceeding for DC voltage stress does not exist. This implies the development and evaluation of alternative and suitable basic approaches. Although diverse promising approaches are identified, recent research is focused on standard atmospheric conditions. Due to the fact that this is just partly consistent to real operating conditions, additional research is required. Focusing on the time domain analysis of corona discharges, occurring under positive DC voltage stress in air, a measurement method for investigating the influence of varying atmospheric quantities is presented. Measurements are carried out for five different relative humidity levels in the range of $20 \%$ to $95 \%$ and for four different temperature levels in the range of $20{ }^{\circ} \mathrm{C}$ to $65^{\circ} \mathrm{C}$. As characterizing pulse shape parameters, the rise time, the pulse width and the fall time are determined as well as the apparent charge. The gained values are compared to each other and reconciled with physical processes.
\end{abstract}

Index Terms - corona discharge, DC voltage, humidity level, pulse shape parameter, temperature level

\section{INTRODUCTION}

$\mathrm{P}$ ARTIAL discharges are the result of a local electrical stress concentration and are defined as localized discharges, which partially bridge the electrical insulation between

This manuscript was submitted on June $8^{\text {th }}, 2018$ and revised on June $25^{\text {th }}$, 2018 as an extended version of the paper "Influence of humidity on pulse shape parameters of positive corona discharges in air at DC voltage" presented at the $17^{\text {th }}$ International Conference on Environment and Electrical Engineering, Milan/Italy, June 2017 [1].

Ulrich Lühring, Daniel Wienold and Frank Jenau are with the Institute of High Voltage Engineering, TU Dortmund University, Dortmund, Germany (e-mail: ulrich.luehring@ tu-dortmund.de).

Supported by:

Cog | Federal Ministry for Economic Affairs and Energy

on the basis of a decision by the German Bundestag conductors [2]. Due to the damaging effect on the condition of isolation, the partial discharge (PD) diagnosis is a key component of electrical diagnostic procedures. In addition to a registration of local weak points, the focus of measurement and analysis techniques is in particular on the identification of the location and the type of fault. Regarding the latter, it is taken advantage of the fact that the appearance of PDs depends on various factors, which are influenced by the type of fault. Under AC voltage stress the phase resolved pattern is the best established and most commonly used diagnostic tool to distinguish between different types of fault [3]. This diagnostic procedure analyzes the link between discharge pulses and the phase angle of their occurrence with respect to the test voltage. The application is consequently restricted to $\mathrm{AC}$ voltage and not transferable to $\mathrm{DC}$ voltage. A similar recognized diagnostic tool for DC voltage stress does not exist.

The increasing importance of DC transmission systems requires the development and evaluation of alternative and suitable basic approaches. Therefore, the PD diagnosis at DC voltage stress is subject of current research, whereby diverse promising basic approaches could be identified. Regarding measurements based on the conventional PD measuring circuit, experimental tests indicate that an estimation of the magnitude of PDs [4], an analysis of the time lag between subsequent PD impulses [5] and the time domain analysis [6] are appropriated for a precise defect classification. In addition to that, unconventional measuring methods, such as the capturing of the electromagnetic spectrum by using a broadband antenna, are increasingly moving to the fore. With respect to DC applications it is e.g. shown in [7] and [8], that an evaluation of acquired data of the electromagnetic spectrum of PDs in the frequency domain respectively in the time domain is suited to distinguish between different types of fault.

An advantage of the time domain analysis is the direct relationship between the pulse shape of the PD impulse current and the causing processes. Furthermore, it is well known that the electromagnetic spectrum of PDs depends on the pulse shape of the discharge current [9]. The time domain analysis focusses on parameters, which are directly obtained from the pulse shape. The rise time, the pulse width and the fall time as primary parameters are considered as well as Ministry for Economic Affairs and Energy (BMWi)

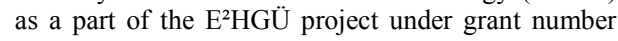
$03 \mathrm{ET} 7514$. 
secondary parameters such as the coefficient of variation, the kurtosis or the skewness. A comparative analysis of these parameters, gained from PDs of idealized and artificial defects under DC voltage stress, outlines the possibility to differentiate between different types of fault [10] and defects in varying insulating media [11], [12]. Extensive experimental investigations and statistical evaluations, presented in [6], verify that the time domain analysis of PDs enables a precise defect classification. Comparative investigations of corona discharges occurring under $\mathrm{AC}$ and $\mathrm{DC}$ voltage stress point out, that the associated pulse shape parameters are not affected by the voltage waveform [13]. Based on these findings [14] demonstrates the general suitability of the time domain analysis to identify the type of fault at AC voltage as well.

The abovementioned investigations have in common that they are comprehensively realized under ambient atmosphere, which approximately corresponds to the reference atmosphere, and under a voltage stress which complies with the partial discharge inception voltage or a predefined voltage level above it. A variation of these parameters is not taken into consideration, which is just partly consistent with real operating conditions. Whereas detailed information regarding an influence of the test voltage level are outlined in [15], additional research concerning the influence of varying atmospheric parameters, such as the air pressure, the humidity level and the temperature level, on the appearance of PDs has to be carried out. Studies in relation to an influence of the gas pressure are outlined in [16] and [17]. Although detailed information from the presented measurement data are not derivable, these investigations indicate increasing discharge amplitudes and decreasing pulse widths as well as decreasing fall times if the gas pressure is raised. A consideration of a varying temperature and humidity level focuses on investigations regarding the PD intensity, the discharge inception voltage and the breakdown voltage. For example, [18] respectively [19] evince a slight decrease of the PD inception voltage and the breakdown voltage, if the temperature level is raised. Concerning a rising humidity level, a decreasing PD inception voltage and an increasing breakdown voltage is pointed out in [20] respectively [21]. This indicates a dependency between the partial discharge impulse current and the temperature level as well as the humidity level.

The purpose of this contribution is to investigate the influence of the temperature and the humidity level on the pulse shape by focusing on positive corona discharges in air. Therefore, corona discharges are generated by using a needleto-plate set-up. The latter is part and parcel of an optimized measuring circuit. An appropriate measuring method is presented and applied to capture the pulse shape of corona discharges. To characterize the pulse shapes the rise time, the pulse width and the fall time are determined as well as the apparent charge for PDs occurring at temperature levels of $20^{\circ} \mathrm{C}, 35^{\circ} \mathrm{C}, 50{ }^{\circ} \mathrm{C}$ and $65^{\circ} \mathrm{C}$ and for relative humidity levels of $20 \%, 40 \%, 60 \%, 80 \%$ and $95 \%$.

\section{EXPERIMENTAL SET-UP}

An accurate determination of pulse shape parameters presupposes a broadband measuring system, consisting of a measuring circuit and a measuring instrument. In [22] an overall bandwidth of at least $1 \mathrm{GHz}$ is demanded to capture initial pulse shapes, which are not impaired by reflection and refraction processes. If this requirement is fulfilled, detailed information regarding the causing and influencing processes can be gained.

\section{A. PD measuring circuit}

The measurements are implemented by using a basic PD measuring circuit, which is in accordance to IEC 60270. As shown in Fig. 1, the PD measuring circuit consists of a coupling capacitor $\mathrm{C}_{\mathrm{c}}$, a measuring cell with a needle-to-plate set-up (DUT), a measuring impedances $Z_{\mathrm{m} 1}$ respectively $Z_{\mathrm{m} 2}$ and a decoupling impedance $Z_{d}$. The latter decouples the discharge current circuit from a high voltage power supply $\mathrm{U}_{0}$, which provides a positive DC voltage with a low residual ripple. To determine the $\mathrm{PD}$ inception voltage an external quadripole of a PD measuring system is set as $Z_{\mathrm{m} 1}$ in series to $C_{\mathrm{c}}$. The pulse shapes are captured with a broadband oscilloscope, whose input resistor is set as $Z_{\mathrm{m} 2}$ in series to the DUT.

\section{B. Measuring Cell}

A decoupling of the partial discharge impulse current from the weak point to the broadband oscilloscope devoid of distortions is enabled by using a measuring cell optimized from a high voltage and high frequency point of view. Core component of the measuring cell, whose sectional view is shown in Fig. 2, is a conical transmission line with a constant line impedance of approximately $50 \Omega$. The suitability of such a conical transmission line for an accurate measurement of the pulse shape is proven in [11] and [23]. In total four air inlets, which are arranged radially in the same height, ensure that the temperature and the humidity level within the measuring cell can comply with the external levels.

\section{Test device}

To generate corona discharges a sharp needle is attached to the cylindrical high voltage electrode. The air clearance between the ground electrode and the needle tip is set to $15 \mathrm{~mm}$.

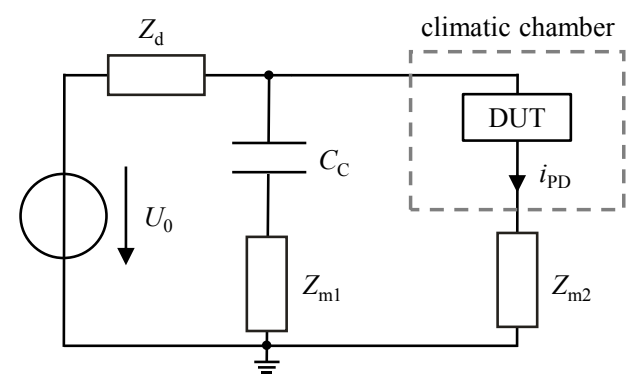

Fig. 1. PD measuring circuit according to IEC 60270 to determine the PD inception voltage and to capture the pulse shape. 


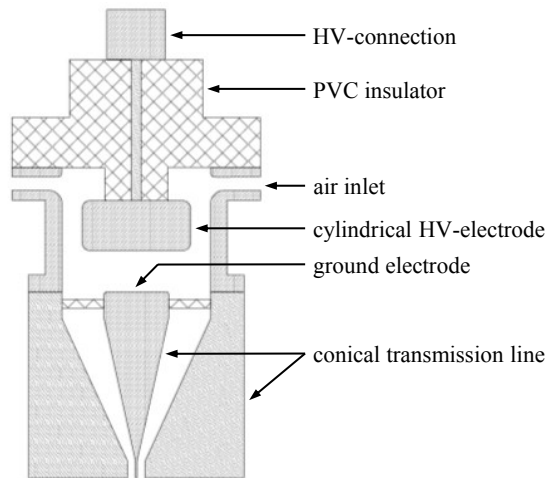

Fig. 2. Sectional view of the measuring cell optimized from a high voltage and high frequency point of view.

\section{EXPERIMENTAL PROCEDURE}

The influence of the temperature level and the humidity level on the pulse shape of positive corona discharges is investigated by considering different climatic conditions. As shown in in Fig. 313 different climatic conditions are considered, whereby the temperature level is varied in the range of $20^{\circ} \mathrm{C}$ to $65^{\circ} \mathrm{C}$ and the relative humidity level is varied in the range of $20 \%$ to $95 \%$. A variation of these is made possible by placing the measuring cell in a climatic chamber, which is presented in [24]. The climatic chamber is characterized by a high long-term stability and an accurate regulation of the temperature and the relative humidity level.

To exclude an influence of the test voltage level, which occurs if the test voltage is raised above the inception voltage [15], all measurements are executed at the respective PD inception voltage. The latter is ascertained in advance for each climatic condition.

\section{A. Determination of the inception voltage}

Up to now, the term of the PD inception voltage under DC voltage stress is not clearly defined. In the framework of this investigation the PD inception voltage is determined by

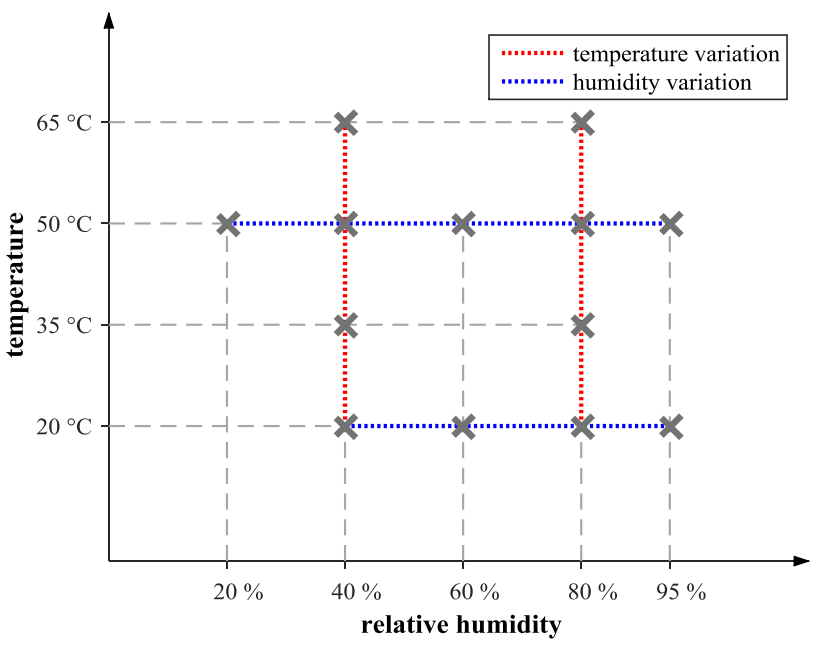

Fig. 3. Overview of the investigated climatic conditions.

increasing the test voltage in steps of $0.5 \mathrm{kV}$ starting from zero. As shown in Fig. 4 the test voltage is enhanced by additional $0.5 \mathrm{kV}$, if PDs are not detected within one minute.

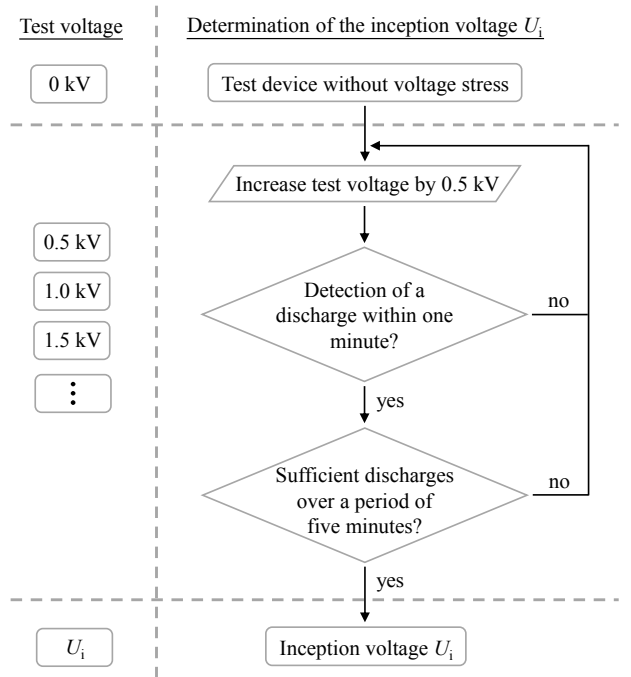

Fig. 4. Flow diagram for the determination of the PD inception voltage

If sufficient PDs are measurable at a constant test voltage level over a period of five minutes, the PD inception voltage is assumed to be reached.

\section{B. Capturing of the pulse shapes}

The climatic conditions are successively provided by the climatic chamber, whereby special attention is paid in order to prevent the formation of condensate. In the case of a sole variation of the humidity level, an additional residence time of at least two hours is taken into consideration before performing a measurement after having reached a stationary state in the climatic chamber. If the temperature level is changed, the additional residence time is set to at least 12 hours. This ensures the desired climatic conditions within the measuring cell.

Previous investigations demonstrate that the first determinable pulse shapes reach larger amplitudes than the following [25], [26]. Due to these findings, the capturing of 100 sequent pulse shapes for each climatic condition is started after a stress duration of ten minutes. Furthermore, this ensures that the PDs are not mainly energized by the capacitive field [5].

\section{DATA ANALYSIS AND PRESENTATION}

The captured pulse shapes are characterized by the determination of the rise time ( $20 \%$ to $80 \%$ ), the pulse width ( $50 \%$ to $50 \%$ ), the fall time ( $80 \%$ to $20 \%$ ) and the apparent charge.

To present and compare the location and the distribution of the determined pulse shape parameters, box plots are used. This kind of graphic representation, which is among others shown in Fig. 5, divides the entire range of values into four subsets. The median is represented by the bar within the box, which itself contains the middle $50 \%$ of all determined values. The boundaries of the box are the $25 \%$ quartile and the $75 \%$ quartile. Their distance delineates the interquartile range. Values, having a greater distance to the box than 1.5 times of the interquartile range, are designated as outliers and are characterized by a plus symbol. 


\section{RESULTS AND DISCUSSION}

\section{A. Appropriateness of the measurement method}

The appropriateness of the experimental procedure presented in section III is evaluated by investigating selected climatic conditions more than once. To realize the different measurement series the climatic condition within the measuring cell is adjusted anew and the needle, which is attached to the high voltage electrode, is exchanged. In addition to that a long-term study is executed to examine the temporal development of the considered pulse shape parameters. In dependence on the reference atmosphere, these measurements are comprehensively carried out at a temperature level of $20^{\circ} \mathrm{C}$ and an absolute humidity level of $11 \mathrm{~g} / \mathrm{m}^{3}$.

In terms of two different measurement series, the box plots for 100 values of the determined pulse shape parameters are exemplarily shown in Fig. 5. Regarding the median the rise time evinces with approximately $10 \%$ the largest deviation of all considered pulse shape parameters. In contrast, the deviation of the associated median values of the pulse width and the fall time are smaller than $5 \%$ and therefore very low.

The temporal development of the pulse shape parameters, determined from the captured pulse shapes in a long-term study over a period of 360 minutes, is shown in Fig. 6. In the case of the rise time, the maximum deviation of all identified medians from that after a stress duration of ten minutes amounts to $5 \%$. For the pulse width and the fall time the maximum deviation is smaller than $1 \%$. A consideration of the illustrated temporal development clarifies that a significant decrease of the medians after a stress duration of 10 minutes is not ascertainable.

Accordingly, the presented measurement method is suited to investigate the influence of the temperature and the humidity level on pulse shape parameters with a high repeatability, if the defined stress duration is obeyed before starting the data acquisition.

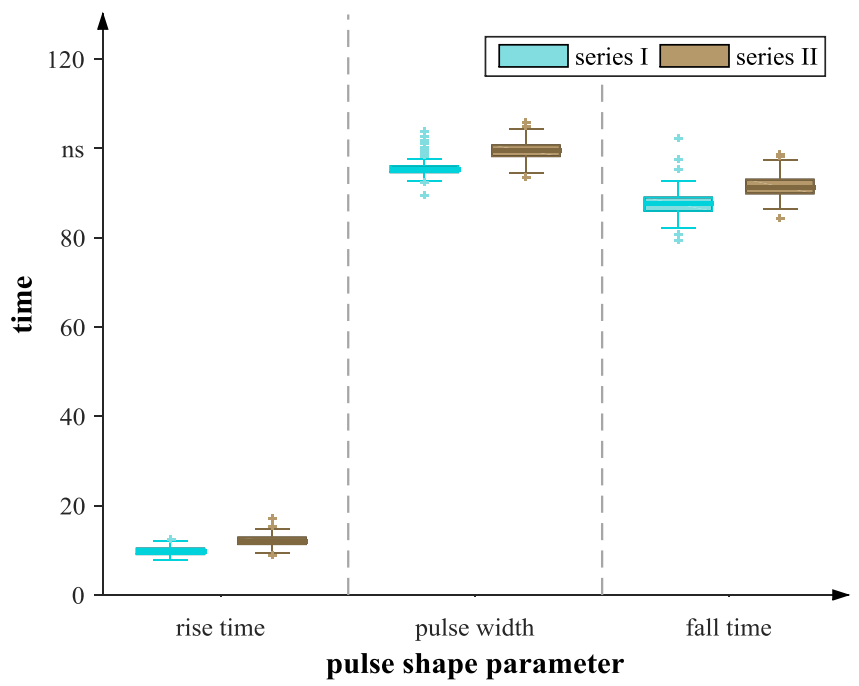

Fig. 5. Box plots for the pulse shape parameters rise time, pulse width and fall time of two different measurement series carried out at a temperature level of $20^{\circ} \mathrm{C}$ and a absolute humidity level of $11 \mathrm{~g} / \mathrm{m}^{3}$.

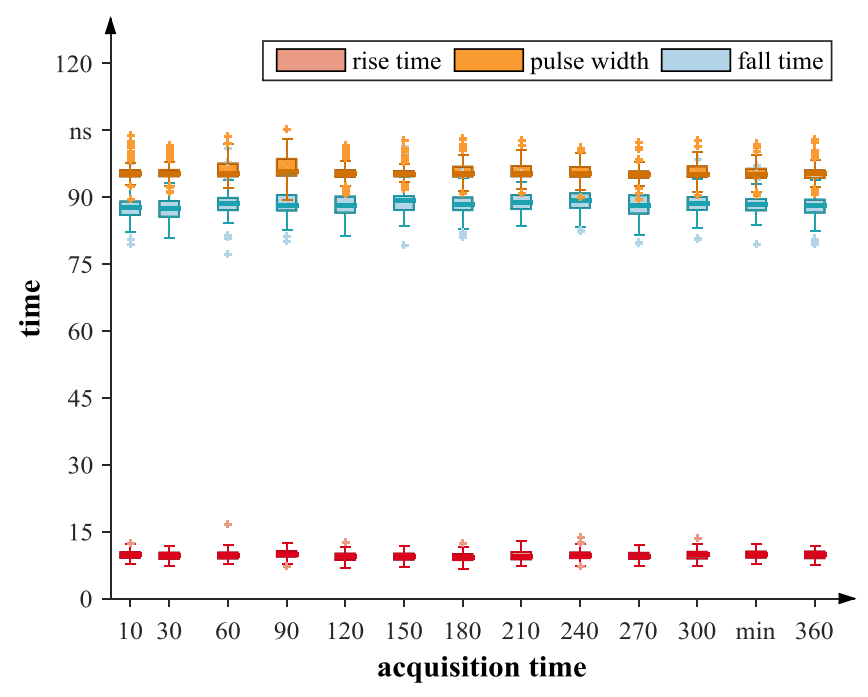

Fig. 6. Temporal development of the the pulse shape parameters rise time, pulse width and fall time as part of a long-term study carried out at a temperature level of $20^{\circ} \mathrm{C}$ and an absolute humidity level of $11 \mathrm{~g} / \mathrm{m}^{3}$.

\section{B. Influence of the absolute humidity level}

A consideration of the influence of a varying absolute humidity on the pulse shape parameters enables a simultaneous consideration of the temperature level and the relative humidity level. As stated in [27] a conversion is feasible according to equation (1).

$$
A H=\frac{6.11 \cdot R H \cdot e^{\frac{17.6 \cdot T}{243+T}}}{0.4615 \cdot(273+T)}
$$

$A H: \quad$ absolute humidity level in $\mathrm{g} / \mathrm{m}^{3}$

$R H: \quad$ relative humidity level in percent

$T$ : $\quad$ temperature level of ambient air in ${ }^{\circ} \mathrm{C}$

Fig. 7 exemplarily shows the medians for the pulse width and the fall time in dependence on the absolute humidity. It should be noted that the fundamental curve progression is identical for all considered temperature levels. This indicates that the absolute humidity level is the decisive influencing factor, whereby an additional influence of the temperature level on the pulse shape parameters cannot be excluded.

\section{Influence of the temperature level}

In Fig. 8 the box plots for 100 values of the determined pulse shape parameters rise time, pulse width and fall time are depicted as well as those for the apparent charge for positive corona discharges occurring at a varying temperature level. It is shown, that a variation of the temperature level affects the pulse shape for both considered relative humidity levels. All determined pulse shape parameters decrease with an increasing temperature level. This is particularly apparent in the case of the fall time and the pulse width. Focusing on the latter and on the measurements at a relative humidity level of $40 \%$, the median for a temperature level of $20{ }^{\circ} \mathrm{C}$ is in the range of $130 \mathrm{~ns}$ whereas the median for a temperature level of $65^{\circ} \mathrm{C}$ is in the range of $22 \mathrm{~ns}$. This corresponds to a reduction of almost one sixth. 

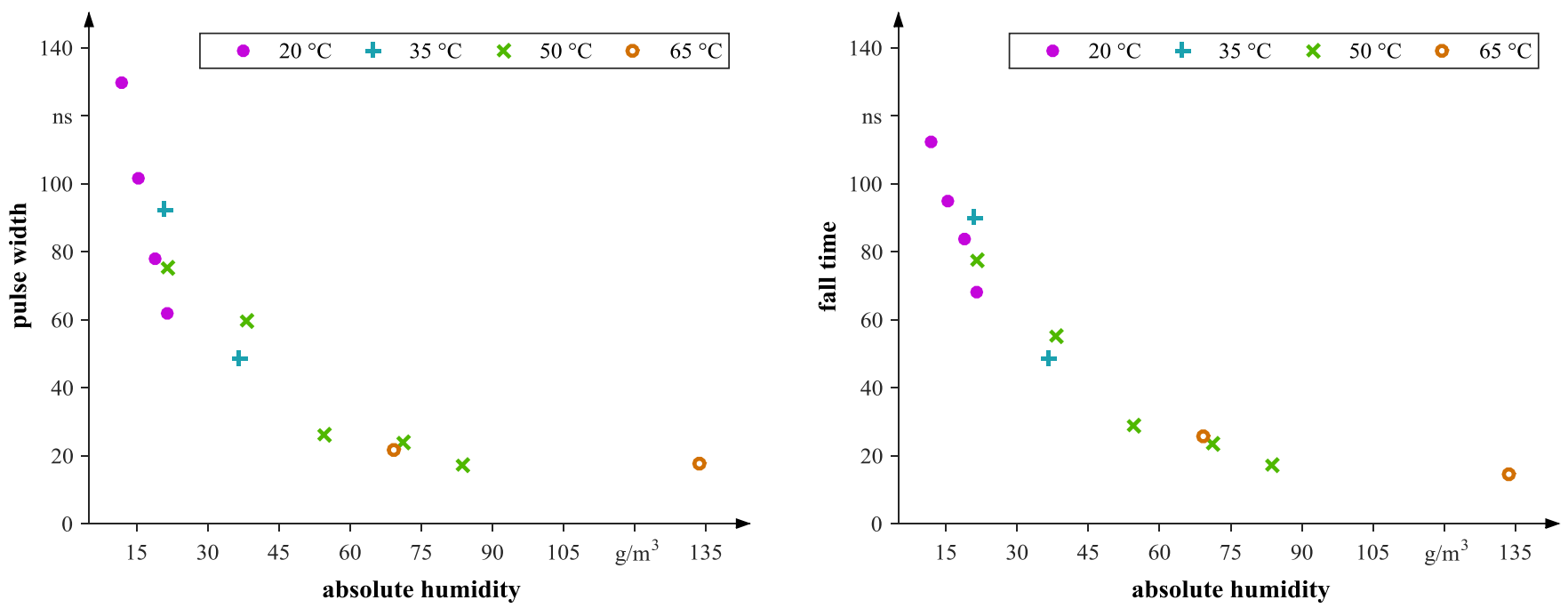

Fig. 7. Medians of the pulse width (left) and the fall time (right) for positive corona discharges in dependence of the absolute humdity.
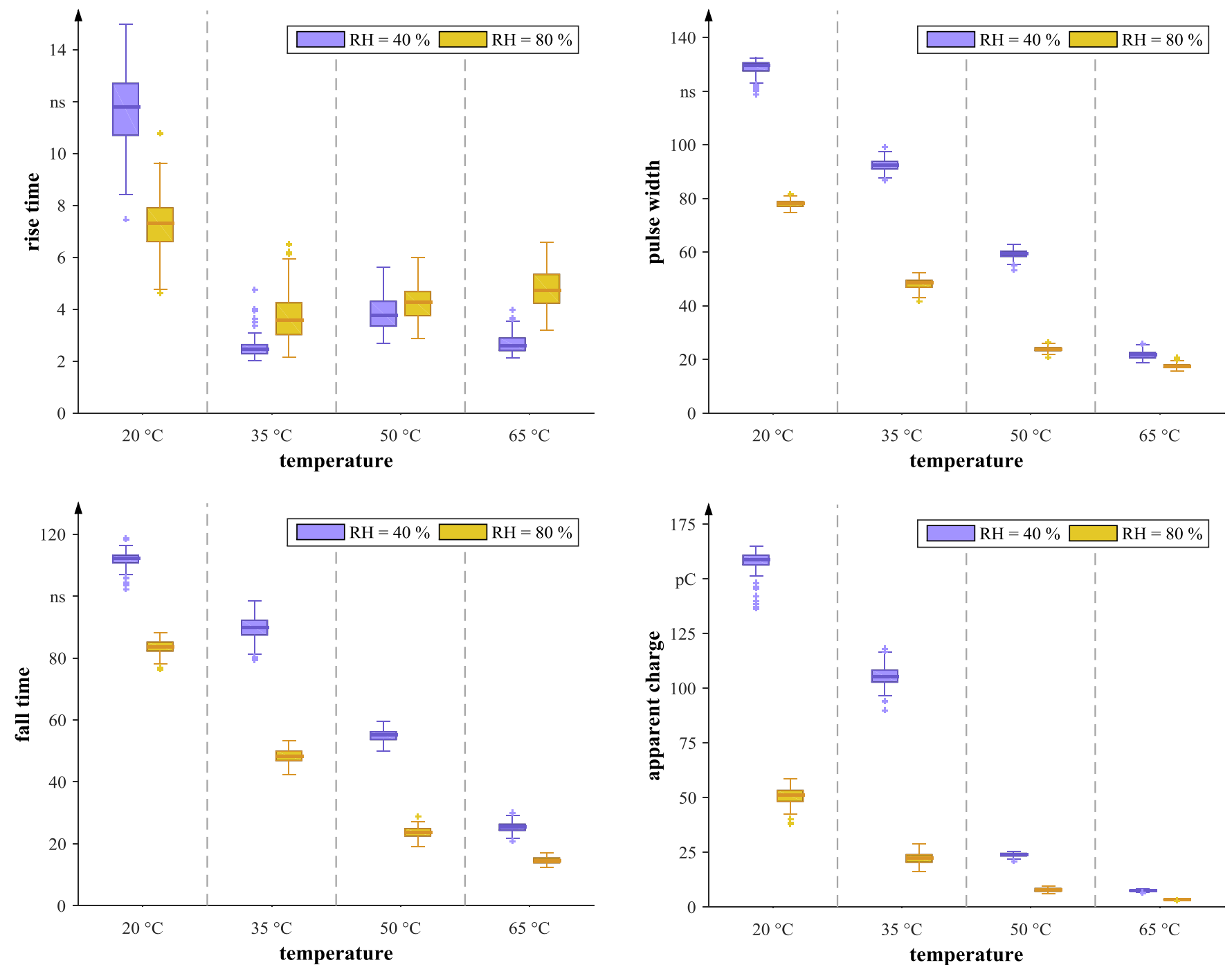

Fig. 8. Box plots for the pulse shape parameters rise time (top left), pulse width (top right), fall time (bottom left) and the apparent charge (bottom right) of positive corona discharges occuring under a varying temperature level for relative humidity levels of $40 \%$ and $80 \%$. 


\section{Influence of the relative humidity level}

The box plots for the determined values concerning an influence of the relative humidity level are illustrated in Fig. 9. The distribution and the location of the 100 gained values for the rise time, the pulse width, the fall time and the apparent charge clarifies that the pulse shape is affected by a varying relative humidity level. With the exception of the rise time, all considered parameters decrease with an increasing relative humidity level. In relation to the pulse shape parameters this is particularly apparent in the case of the pulse width and the fall time. Focusing on the fall time and on the measurements at $50{ }^{\circ} \mathrm{C}$, the median for a relative humidity level of $20 \%$ is in the range of $77 \mathrm{~ns}$ whereas the median for a relative humidity level of $95 \%$ is in the range of $17 \mathrm{~ns}$. This corresponds to a reduction of almost one fifth. Besides the fundamental influence of the relative humidity level on the pulse shape parameters, the box plots, illustrated in Fig. 9, suggest a linear dependency between the location of the pulse shape parameters and the relative humidity level for the measurements at $20{ }^{\circ} \mathrm{C}$. For the measurements at $50^{\circ} \mathrm{C}$ the
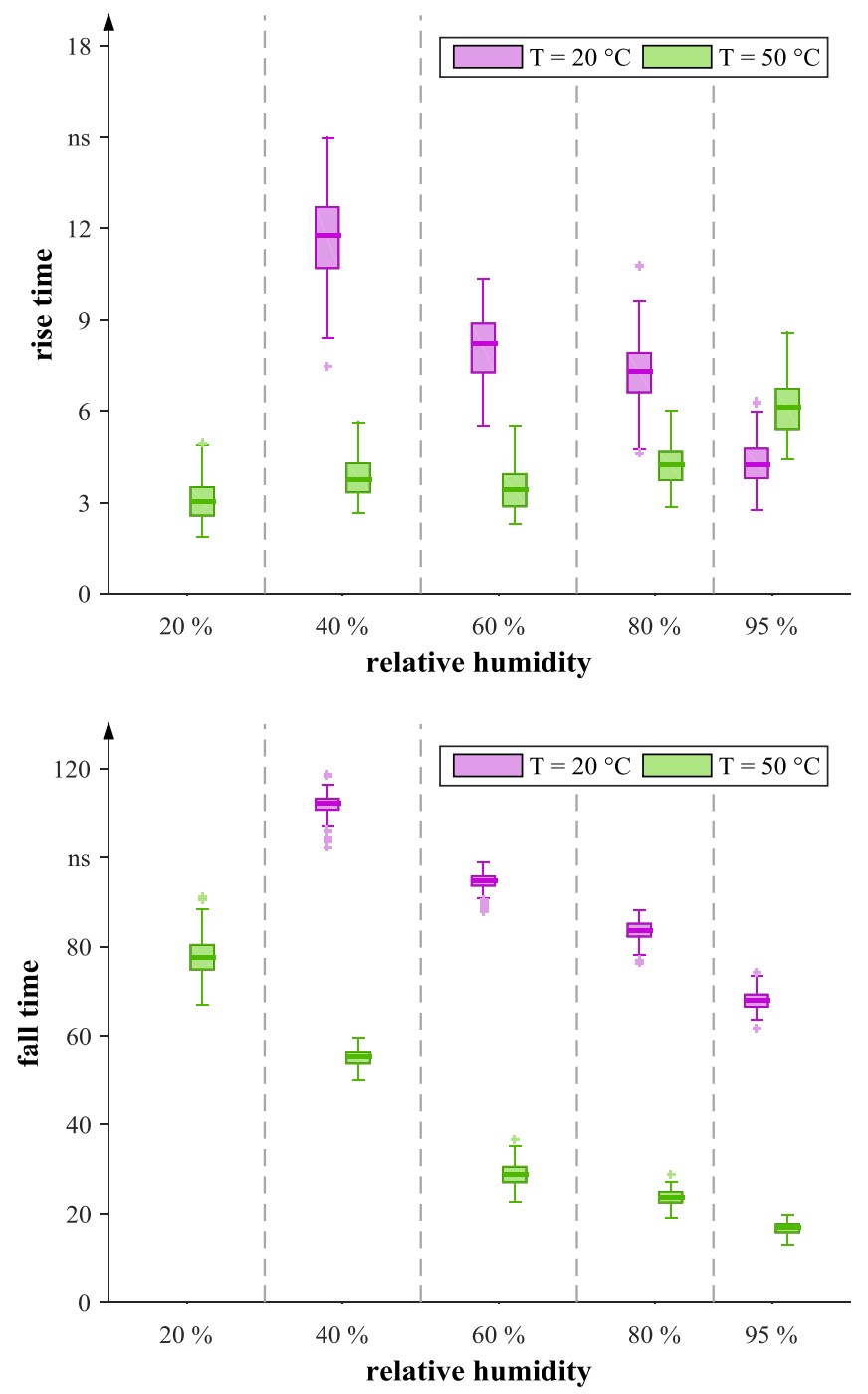

change in value suspects a non-linear relationship. This indicates that the relative humidity level is not the decisive influencing factor.

\section{E. Reconciliation with physical processes}

Independent of the considered climatic condition, the captured pulse shapes are characterized by a steep ascent and a slower descent. Relating to [28] this fundamental curve progression is directly traceable to involved physical processes. The needle-to-plate setup generates a strongly inhomogeneous electric field. Prerequisite for the development of a partial discharge process is the availability of a free electron in the area of the highest electric field strength. In the current case of a needle, appearing as positive high voltage electrode, the free electron is accelerated into the area of increasing field strength. This is associated with an energy absorption. Collisions with gas molecules lead to the emergence of secondary electrons and positive ions if the ionization energy is reached. A continued existence of the necessary ionization condition results in an avalanche effect, which causes the steep ascent of the pulse shape. An increase
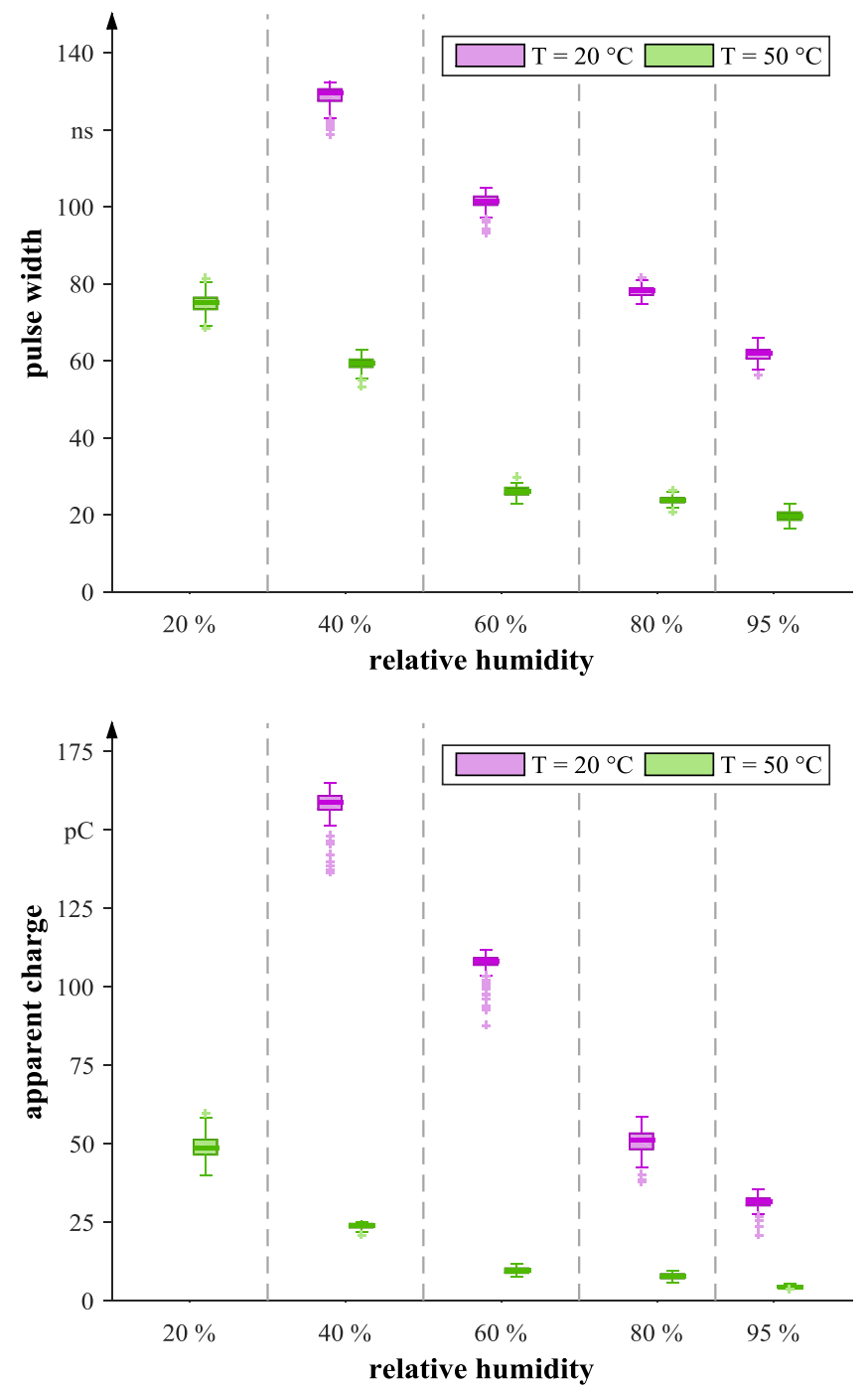

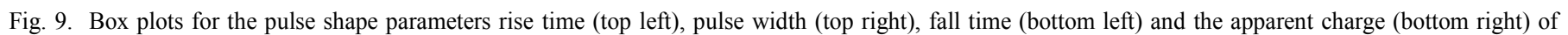
positive corona discharges occuring under a varying relative humidity level for temperature levels of $20^{\circ} \mathrm{C}$ and $50{ }^{\circ} \mathrm{C}$. 
of the mean free path, e.g. by reducing the air density as a result of a rising temperature level, favors this effect. Whereas the electrons move very rapidly towards the needle tip and are accepted by the anode or attached to molecules with a high electron affinity, the positive ions form a positive space charge. Because of the low mobility of the positive ions, the space charge drifts very slowly in the direction of the cathode. This entails a reduction of the effective field strength near the needle tip, which hampers the ionization process, favors the attachment process and leads to a decreasing discharge current. Therefore, the characteristic of the pulse shape is significantly dependent on the ionization and the attachment coefficient

Experimental tests prove that a surge of the humidity level goes along with an increasing attachment coefficient [29] and an increasing ionization coefficient [30]. The same applies to a rise of the temperature level [31]. This knowledge is already utilized to explain the impact of the temperature and the humidity level on the breakdown voltage and the PD inception voltage. Coupled with the fact that the mean free path is magnified, which favors relevant collision processes, a declaration of the ascertained influence of a varying temperature and humidity level on the pulse shape parameters is enabled.

Considering the temporal progression of the PD process described above, an increasing ionization coefficient results in a steeper ascent of the pulse shape and a decreasing rise time. Apart from a growing number of free electrons, the hampering positive space charge is build up in a shorter time span. This is accompanied by a reduction of the pulse width and the amplitude. The minimization of the effective field strength near the anode enhances the possibility of attachment processes. These are favored by an upward attachment coefficient, which results in a decreasing fall time. Consequently, the apparent charge decreases as well, if all abovementioned parameters decline.

\section{CONCLUSIONS}

To obtain detailed information regarding the influence of the temperature and the humidity level, the pulse shapes of corona discharges are captured for 13 different climatic conditions. By performing a long-term study and carrying out investigations at one climatic condition more than once, it is proven that the proposed measuring method is suited to investigate the influence of the temperature and the humidity level. Regarding the latter, a determination of the pulse shape parameters results in comparable values, so that a high repeatability is achieved. This applies also if the needle, which emulates corona discharges, is substituted. A comparative analysis of pulse shape parameters, gained from measurements at different climatic conditions, illustrates that an increasing temperature level at a constant relative humidity level as well as an increasing humidity level results in decreasing pulse shape parameters. Therefore, the interpretation of PD impulses requires a consideration of the climatic condition. The dependency can be reconciled with the reliance of the ionization coefficient and the attachment coefficient on the varying atmospheric parameter. Regarding the influence of the humidity level it is assumed that the absolute humidity is the decisive influencing factor. To verify this assumption, it is recommended to carry out supplementary investigations. This should entail an investigation of a varying temperature level at a constant absolute humidity.

\section{REFERENCES}

[1] U. Lühring, D. Wienold and F. Jenau, "Influence of humidity on pulse shape parameters of positive corona discharges in air at DC voltage", $17^{\text {th }}$ International Conference on Environment and Electrical Engineering, Milan/Italy, June 2017.

[2] IEC 60270:2000, "High-voltage test techniques - Partial discharge measurement (IEC 60270:2000); German version EN 60270:2001”, DIN Deutsches Institut für Normung e.V. und VDE Verband der Elektrotechnik Elektronik Informationstechnik e.V., Berlin, 2001.

[3] D. König and Y. N. Rao, "Partial discharges in electrical power apparatus", Berlin: VDE-Verlag, 1993.

[4] R. S. Bever and J. L. Westrom, "Partial Discharge Testing Under Direct Voltage Conditions", IEEE Transactions on Aerospace and Electronic Systems, Vol. AES-18, No. 1, January 1982.

[5] U. Fromm, "Interpretation of Partial Discharges at dc Voltages", IEEE Transactions on Dielectrics and Electrical Insulation, Vol. 2, No. 5, October 1995.

[6] T. Vogt, "Teilentladungsdiagnose bei Gleichspannung", Dissertation Technische Universität Dortmund, 2015.

[7] D. Wienold, U. Lühring and F. Jenau, "Detection and distinction of partial discharges in air at DC voltage by using a non-conventional approach in the high-frequency range“" $17^{\text {th }}$ International Conference on Environment and Electrical Engineering, Milan/Italy, June 2017.

[8] D. Wienold, U. Lühring and F. Jenau, "Data analysis and verification for DC PD measurements in the high frequency range", $2^{\text {nd }}$ IEEE International Conference on Dielectrics, Budapest/Hungary, 2018, in press.

[9] T. Mutakamihigashi, R. Sakurai, S. Okada and H. Ueno, "Relationship between electric field strength and characteristic frequency in partial discharge", $18^{\text {th }}$ International Symposium on High Voltage Engineering, Seoul/Korea, August 2013.

[10] T. Klueter, J. Wulff, F. Jenau and D. Wienold, "Evaluation of Surfaceand Corona Discharges at DC Voltage", $13^{\text {th }}$ International Conference on Environment and Electrical Engineering, Wroclaw/Poland, November 2013.

[11] T. Klueter, J. Wulff and F. Jenau, "Time Domain Analysis of Partial Discharges at DC Voltage in Air and Insulation Oil", $12^{\text {th }}$ International Conference on Environment and Electrical Engineering, Wroclaw/Poland, May 2013.

[12] T. Klueter, J. Wulff and F. Jenau, "Measurement and Statistical Analysis of Partial Discharges at DC Voltage", $48^{\text {th }}$ International Universities' Power Engineering Conference UPEC 2013, Dublin/Ireland, September 2013.

[13] U. Lühring, D. Wienold and F. Jenau, "Comparative investigation on pule shape parameters of partial discharges in air under AC and DC voltage stress", $51^{\text {st }}$ International Universities' Power Engineering Conference UPEC 2016, Coimbra/Portugal, September 2016.

[14] U. Lühring, D. Wienold and F. Jenau, "Investigation on the Applicability of the Time Domain Analysis of Discharges in Gases for the Defect Identification at AC Voltage", Transactions on Environment and Electrical Engineering, Vol. 2, No. 1, January 2017.

[15] U. Lühring, D. Wienold and F. Jenau, "Investigation on the pulse shape of DC corona discharges in air under varying test voltage level", $2^{\text {nd }}$ IEEE International Conference on Dielectrics, Budapest/Hungary, July 2018, in press.

[16] D. A. Scott and G. N. Haddad, "Negative point-to-plane corona pulses in oxygen", Journal of Physics D: Applied Physics 19, January 1986.

[17] X. Liu, D. G. Kasten and S. A. Sebo, "Partial Discharge Measurements for a Twisted Pair of Insulated Conductors at Low Pressures in Air, Argon and Helium“, IEEE International Symposium on Electrical Insulation, June 2006.

[18] M. Abdel-Salam and N. L. Allen, "Current-voltage characteristics of corona in rod-plane gaps as influenced by temperature", IEE Proceedings - Science, Measurement and Technology, Vol. 150, No 3, May 2003. 
[19] N. L. Allen, M. Abdel-Salam and I. Cotton, "Effects of temperature and pressure change on positive corona and sparkover under direct voltage in short airgaps", IET Science, Measurement \& Technology, Vol. 1, No. 4, July 2007.

[20] X. Bian, L. Wang, J. M. K. MacAlpine and Z. Guan, "Positive Corona Inception Voltages and Corona Currents for Air at Various Pressures and Humidities", IEEE Transaction on Dielectrics and Electrical Insulation, Vol. 17, No. 1, February 2010.

[21] E. Kuffel, "Influence of humidity on the breakdown voltage of spheregaps and uniform-field gaps", Proceedings of the IEE, Paper 3322M, February 1961.

[22] P. Morshuis, "Assessment of dielectric degradation by ultrawide-band PD detection", IEEE Transactions on Dielectrics and Electrical Insulation, Vol. 2, No. 5, October 1995.

[23] M. Kurrat and D. Peier, "Wideband measurement of partial discharges for fundamental diagnostics", $7^{\text {th }}$ International Symposium on High Voltage Engineering, Dresden/Germany, August 1991.

[24] R. Schmerling, M. Freiburg, F. Jenau, T. Weissgerber and F. Pohlmann, „Development and application of a climate chamber for testing large dimensioned high voltage components up to $100 \mathrm{kV}$ ", $18^{\text {th }}$ International Symposium on High Voltage Engineering, Seoul/Korea, August 2013.

[25] H. Okubo and N. Hayakawa, "A novel technique for partial discharge and breakdown investigation based on current pulse waveform analysis", IEEE Transactions on Dielectrics and Electrical Insulation, Vol. 12, No. 4, August 2005.

[26] K. Nanao, Y. Murakami and M. Nagao, "Analysis of Internal Partial Discharge Based on PD Current Waveform", Annual Report: Conference on Electrical Insulation and Dielectric Phenomena, October 2008.

[27] IEC 60060-1:2010, "High-voltage test techniques - Part 1: General definitions and test requirements (IEC 60060-1:2010); German version EN 60060-1:2010”, DIN Deutsches Institut für Normung e.V. und VDE Verband der Elektrotechnik Elektronik Informationstechnik e.V., Berlin, 2011.

[28] L. B. Loeb, "Electrical coronas - Their basic physical mechanism", Berkeley and Los Angeles: University of California Press, 1965.

[29] E. Kuffel, "Electron Attachment Coefficients in Oxygen, Dry Air, Humid Air and Water Vapour", Proceedings of the Physical Society, Vol. 74, No. 3, April 1959.

[30] W. Bauke, "Über den Einfluß des Wasserdampfgehaltes der Luft auf den elektrischen Durchschlag im inhomogenen Feld bei Wechselspannung", Dissertation Technische Universität Berlin, 1968.

[31] W. S. Zaengl, S. Yimvuthikul and G. Friedrich, "The Temperature Dependence of Homogeneous Field Breakdown in Synthetic Air", IEEE Transactions on Electrical Insulation, Vol. 26, No. 3, June 1991. 